FACTA UNIVERSITATIS (NIŠ)

Ser. Math. Inform. Vol. 34, No 4 (2019), 771-780

https://doi.org/10.22190/FUMI1904771R

\title{
ON A DESIGN FROM PRIMITIVE REPRESENTATIONS OF THE FINITE SIMPLE GROUPS
}

\begin{abstract}
Ali Reza Rahimipour
(C) 2019 by University of Niš, Serbia | Creative Commons Licence: CC BY-NC-ND

Abstract. In this paper we present a design construction from primitive permutation representations of a finite simple group $G$. The group $G$ acts primitively on the points and transitively on the blocks of the design. $\mathrm{T}$ he construction has this property that with some conditions we can obtain $t$-design for $t \geq 2$. We examine our design for fourteen sporadic simple groups. As a result we found a $2-(176,5,4)$ design with full automorphism group $M_{22}$.
\end{abstract}

Keywords. primitive permutation; group; finite simple group; automorphism group.

\section{Introduction}

Designs are interesting combinatorial objects. They have important applications in coding theory and information theory. Constructing combinatorial designs by using finite permutation groups is a well-studied subject. Well-known methods to construct 1-design from primitive permutation representations of finite simple groups were introduced by Moori and Key [10, 11]. Also in [5] a generalization of the construction in [10] was described. Here we present a design construction from primitive representations of a finite simple group. The groups we consider are primitive on the points and transitive on the blocks of constructed designs. In some conditions we can construct $t$-designs for $t \geq 2$. We employ this method on some simple groups and calculate full automorphism groups of constructed designs.

Sporadic simple groups are interesting family of the finite simple groups. Designs that are invariant under sporadic groups or have full automorphism group equal to sporadic groups are very interesting. Some of these designs were presented in $[2,8,10,12,13]$. Here we consider fourteen sporadic groups for our purpose. For these sporadic groups we obtained some designs for which the full automorphism groups are the same as the sporadic group or double cover of that. These constructed

Received May 24, 2019; accepted July 30, 2019

2010 Mathematics Subject Classification. Primary 94c30; Secondary 20D08 
designs from sporadic groups are usually 1-design or 2-design and in some cases groups act primitively on the points and the blocks of the designs.

In Section 2, first we present some preliminary definitions and lemmas which will be used in the proof of our main results. In Theorem 2.1 we give our design construction from primitive permutation representations of a finite simple group. Then some properties of these design are considered. One advantage of our design in comparison with the designs presented in $[5,10,11]$ is that we can determine some conditions to construct $t$-design for $t \geq 2$. In Proposition 2.3 these conditions are determined. Applying this result on some finite simple groups we found some 2 -designs from 1-transitive actions of these simple groups. In Section 3 we describ constructed designs from fourteen sporadic simple groups. Especially we make use of large sporadic simple groups $\mathrm{Co}_{3}$ and $\mathrm{Fi}_{23}$. These groups are full automorphism group of the constructed designs which act primitively on the points and the blocks. In Section 4 we present a 2-(176,5,4) design from Mathieu group $M_{22}$. With the best of knowledge, this design is new and group $M_{22}$ acts primitively on the points and transitively on the blocks of this design. The full automorphism group of this design is isomorphic to $M_{22}$.

\section{Design Construction}

In this paper all groups are assumed to be finite. Our notations are standard and for design are from [3] and for group theory and character theory are from $[6,9]$. For the name and structure of finite simple groups we use the Atlas notation [4]. All computations were done with GAP [14] and Magma [1]. All programs are accessible from the author upon request.

Let $t, \lambda, v$ and $k$ be integers such that $1 \leq t \leq k \leq v$ and $\lambda>0$. Let $X$ be an $v$-set. A $t$ - $(v, k, \lambda)$ design is a pair $D=(X, B)$ such that $B$ is a collection of $k$-subsets of $X$ called blocks such that every $t$-subset of $X$ appears in exactly $\lambda$ blocks. A design is called simple if it has no repeated blocks. All designs in this paper are simple. The design $D$ is called symmetric if the number of points and the blocks are equal.

An automorphism of $D$ is a permutation $f$ on $X$ such that $f(b) \in B$ for each $b \in B$. A group whose elements are automorphism of $D$ is called an automorphism group of $D$. We use $A u t(D)$ to denote the full automorphism group of $D$.

Let $G$ be a finite permutation group acting on a set $X$. The orbit of $x \in X$ is defined as $O(x)=\left\{x^{g} \mid g \in G\right\}$ and the stabilizer subgroup of $x$ is $G_{x}=\{g \in$ $\left.G \mid x^{g}=x\right\}$. It is well-known that $|G|=|O(x)| \cdot\left|G_{x}\right|$. For $g \in G$, the conjugacy class of $g$ is $\operatorname{cl}(g)=\left\{a^{-1} g a \mid a \in G\right\}$. It is well-known that $|G|=|c l(g)| \cdot\left|C_{G}(g)\right|$ such that $C_{G}(g)=\{a \in G \mid a g=g a\}$ is centralizer subgroup of $g$ in $G$.

Let $G$ be a finite group and $H$ be a subgroup of $G$. Assume that $\Omega$ is the set of all conjugates of $H$ in $G$. Let $\chi_{H}=\chi(G \mid H)$ be the permutation character corresponding to the action of $G$ on $\Omega$. For $g \in G$ if $c l(g) \cap H=\varnothing$ then $\chi_{H}(g)=0$.

In what follows we present some lemmas that are used in constructing design. 
Lemma 2.1. [6, Corollary 1.5A] Let $G$ be a group acting transitively on a set $\Omega$ with at least two points. Then action of $G$ on $\Omega$ is primitive if and only if for each $x \in \Omega, G_{x}$ is a maximal subgroup of $G$.

Lemma 2.2. [7, Corollary 3.1.3] Let $G$ be a finite group and $H$ a subgroup of $G$ containing a fixed element $x$. Then the number $h$ of conjugates of $H$ in $G$ containing $x$ is given by

$$
h=\left[N_{G}(H): H\right]^{-1} \sum_{i=1}^{m} \frac{\left|C_{G}(x)\right|}{\left|C_{H}\left(x_{i}\right)\right|}
$$

where $x_{1}, \ldots, x_{m}$ are representatives of $H$-conjugacy classes that fuse to the $G$-class $\operatorname{cl}(x)$.

Lemma 2.3. [9, Corollary 5.14] Let $G$ be a finite group and $H$ be a subgroup of $G$. Let $\Omega$ be the set of all conjugates of $H$ in $G$. Then for all $g \in G, \chi_{H}(g)$ is equal to the number of points in $\Omega$ fixed by $g$.

Let $G$ be a finite simple group and $M$ be a maximal subgroup of $G$. Let $\Omega$ be the set of all conjugates of $M$ in $G$. In the action of $G$ on $\Omega$, the stabilizer subgroup of each point of $\Omega$ is conjugate to $M$. For $g \in G$ let $\operatorname{cl}(g) \cap M \neq \varnothing$. Then by Lemma 2.2 each $g \in G$ is in $r=\sum_{i=1}^{m} \frac{\left|C_{G}(g)\right|}{\left|C_{M}\left(g_{i}\right)\right|}$ conjugates of $M$, where $g_{1}, \ldots, g_{m}$ are representatives of the $M$-conjugacy classes that fuse to the $G$-class $c l(g)$. Then by Lemma 2.3, $\chi_{M}(g)=r$. Let $n X$ be a conjugacy class of the elements of order $n$ such that $n X \cap M \neq \varnothing$. Consider $g \in n X$. Then $g$ is contained in $r$ conjugates of $M$. We define $B_{g, M}=\left\{M_{1}, \ldots, M_{r}\right\}$ such that $M_{i}$ for $i \in\{1,2, \ldots, r\}$, is a conjugate of $M$ for which $g \in M_{i}$. Also set $S_{g, M}=\cap_{i=1}^{r} M_{i}$. Clearly for $g, g^{\prime} \in n X$ if the subgroups $S_{g, M}$ and $S_{g^{\prime}, M}$ are conjugate, then $\left|S_{g, M} \cap n X\right|=\left|S_{g^{\prime}, M} \cap n X\right|$.

Lemma 2.4. Let $G$ be a finite simple group and $M$ be a maximal subgroup of $G$. Let $n X$ be a conjugacy class of the elements of order $n$ such that $n X \cap M \neq \varnothing$. Then for each $g, g^{\prime} \in n X$ the following hold:

1. $B_{g, M}=B_{g^{\prime}, M}$ if and only if $S_{g, M} \cap n X=S_{g^{\prime}, M} \cap n X$,

2. if $S_{g, M} \cap S_{g^{\prime}, M} \cap n X \neq \varnothing$ then $S_{g, M} \cap n X=S_{g^{\prime}, M} \cap n X$,

3. $\frac{|n X|}{\left|S_{g, M} \cap n X\right|}$ is a positive integer,

4. $\frac{|M \cap n X|}{S_{g, M} \cap n X \mid}$ is a positive integer.

Proof. (1) Let $B_{g, M}=B_{g^{\prime}, M}$. Then obviously $S_{g, M} \cap n X=S_{g^{\prime}, M} \cap n X$. Now suppose $S_{g, M} \cap n X=S_{g^{\prime}, M} \cap n X$. Then $g, g^{\prime} \in S_{g, M}$ and $g, g^{\prime} \in S_{g^{\prime}, M}$. If $B_{g, M} \neq B_{g^{\prime}, M}$ without lose of generally, there exists $M^{\prime}$, a conjugate of $M$ such that $M^{\prime} \in B_{g, M}$ but $M^{\prime} \notin B_{g^{\prime}, M}$. Now since $g^{\prime} \in S_{g, M}$ then $g^{\prime} \in M^{\prime}$ and $M^{\prime} \in B_{g^{\prime}, M}$ which is a contradiction. (2) Suppose $x \in S_{g, M} \cap S_{g^{\prime}, M} \cap n X$. So $x \in S_{g, M}$ 
and $x \in S_{g^{\prime}, M}$. Then we have $B_{x, M}=B_{g, M}=B_{g^{\prime}, M}$ and by (1) the result is achieved. (3) By (2) each element of $n X$ is contained in a unique $S_{g, M} \cap n X$. Then $n X=\cup_{i=1}^{k}\left(S_{g_{i}, M} \cap n X\right)$, which is a disjoint union of the elements of class $n X$ for some $S_{g_{i}, M}$ for $i \in\{1,2, \ldots, k\}$. Therefore $k=\frac{|n X|}{\left|S_{g, M} \cap n X\right|}$ is positive integer. (4) According to the proof of (3) we have $n X=\cup_{i=1}^{k}\left(S_{g_{i}, M} \cap n X\right)$. For each $i \in\{1,2, \ldots, k\}, S_{g_{i}, M} \cap M \cap n X=S_{g_{i}, M} \cap n X$ or $S_{g_{i}, M} \cap M \cap n X=\varnothing$. Therefore $n X \cap M=\cup_{j=1}^{h}\left(S_{g_{j}, M} \cap n X\right)$ such that for each $j \in\{1,2, \ldots, h\}$ we have $S_{g_{j}, M} \cap$ $M \cap n X=S_{g_{j}, M} \cap n X$ hence $h=\frac{|M \cap n X|}{S_{g, M} \cap n X}$ is positive integer.

Now we are ready to present a design construction from primitive permutation representations of a finite simple group.

Theorem 2.1. Let $G$ be a finite simple group. Let $M$ be a maximal subgroup of $G$ and $\Omega$ be the set of all conjugates of $M$ in $G$. Let $n X$ be a conjugacy class of the elements of order $n$ such that $M \cap n X \neq \varnothing$ and $g \in n X$. Set $B=\left\{B_{x, M} \mid x \in n X\right\}$. Then $D=(\Omega, B)$ is a,

$$
1-\left([G: M], \chi_{M}(g), \frac{|M \cap n X|}{\left|S_{g, M} \cap n X\right|}\right)
$$

design which has $\frac{|n X|}{\left|S_{g, M} \cap n X\right|}$ blocks. Also $G$ is an automorphism group of $D$ which acts primitively on the points and transitively on the blocks of $D$.

Proof. In the action of $G$ on $\Omega$, the stabilizer of each point of $\Omega$ is conjugate to $M$. Since $B_{g, M}$ is the collection of all conjugates of $M$ that contain $g$, by Lemma 2.3 the length of a block is $\chi_{M}(g)$. Clearly $\left|S_{g, M} \cap n X\right| \geq 1$. If $\left|S_{g, M} \cap n X\right|=1$ then by Lemma 2.4(1) for every $g^{\prime}, g^{\prime \prime} \in n X$ we have $B_{g^{\prime}, M} \neq B_{g^{\prime \prime}, M}$ and so $|B|=|n X|$. If $\left|S_{g, M} \cap n X\right|=m>1$ then by Lemma 2.4(1) for $m$ elements $g_{1}, \ldots, g_{m}$ of $S_{g, M} \cap n X$ we have $B_{g_{1}, M}=B_{g_{2}, M}=\ldots=B_{g_{m}, M}$. So according to the proof of Lemma 2.4(3), $n X=\cup_{i=1}^{k}\left(S_{g_{i}, M} \cap n X\right)$ for $i \in\{1,2, \ldots, k\}$, which is a disjoint union of the elements of $S_{g_{i}, M} \cap n X$. Then for each $S_{g_{i}, M} \cap n X$ there exists a unique block. Hence the number of different blocks is equal to $\frac{|n X|}{\left|S_{g, M} \cap n X\right|}$ which by Lemma 2.4(3) is a positive integer. By the proof of Lemma 2.4(4) $M \cap n X=\cup_{j=1}^{h}\left(S_{g_{j}, M} \cap n X\right)$ for $j \in\{1,2, \ldots, h\}$, which is a disjoint union of the elements of $S_{g_{j}, M} \cap n X$. Then for each $j \in\{1,2, \ldots, h\}$, subgroup $M$ is in a unique block. Then each point appears in exactly $\frac{|M \cap n X|}{\left|S_{g, M} \cap n X\right|}$ blocks which by Lemma 2.4(4) is a positive integer. The group $G$ acts on the points and the blocks with conjugation. Since $M$ is maximal then by Lemma 2.1, G acts primitively on the points. Each block is corresponding to an element of $n X$ and since $G$ is transitive on $n X$ then is transitive on the blocks.

We denote the constructed design in Theorem 2.1 by $D(G, M, n X)$. The design $D(G, M, n X)$ is not necessary symmetric and the action of $G$ on the blocks is not necessary primitive. In the following propositions we consider some conditions to achieve these properties. 
Proposition 2.1. Let $G$ be a finite simple group. Let $M$ be a maximal subgroup of $G$ and $\Omega$ be the set of all conjugates of $M$ in $G$. Let $n X$ be a conjugacy class of the elements of order $n$ such that $M \cap n X \neq \varnothing$ and $g \in n X$. If $C_{G}(g)$ is maximal subgroup then the action of $G$ on the blocks of $D(G, M, n X)$ is primitive.

Proof. Since $C_{G}(g)$ is maximal, by Lemma 2.1 the action of $G$ on right cosets of $C_{G}(g)$ is primitive. Each block is corresponding to an element of $n X$ and respectively to a right coset of $C_{G}(g)$. Then the action of $G$ on the blocks of $D(G, M, n X)$ is primitive.

Let $g \in n X$ and consider $D(G, M, n X)$. According to the definition, for every $x \in C_{G}(g)$ we have $B_{g, M}=B_{g^{x}, M}=\left(B_{g, M}\right)^{x}$. Therefore $C_{G}(g)$ is a subgroup of stabilizer of the block $B_{g, M}$.

Proposition 2.2. Let $G$ be a finite simple group. Let $M$ be a maximal subgroup of $G$ and $\Omega$ be the set of all conjugates of $M$ in $G$. Let $n X$ be a conjugacy class of the elements of order $n$ such that $M \cap n X \neq \varnothing$ and $g \in n X$. Then the following hold,

1. if $C_{G}(g)$ is conjugate to $M$ and $\left|S_{g, M} \cap n X\right|=1$ then $D(G, M, n X)$ is symmetric and $G$ acts on the blocks primitively,

2. if $\left|S_{g, M} \cap n X\right| .\left|C_{G}(g)\right|=|M|$ then $D(G, M, n X)$ is symmetric.

Proof. (1) The number of points and blocks in $D(G, M, n X)$ are $\frac{|G|}{|M|}$ and $\frac{|n X|}{\left|S_{g, M} \cap n X\right|}=$ $\frac{|G|}{\left|S_{g, M} \cap n X\right| \cdot\left|C_{G}(g)\right|}$, respectively. Since $C_{G}(g)$ is conjugate to $M$ then $\left|C_{G}(g)\right|=|M|$ and the number of points and blocks are equal. Also by Proposition 2.1 the action of $G$ on blocks is primitive. (2) In this case clearly the number of points and blocks are equal and $D(G, M, n X)$ is symmetric.

In Table 2.1, 2.2 and 2.3 the columns from left are: number of row, the considered finite simple group $G$, maximal subgroup $M$ of $G$, a conjugacy class of $G$, properties of the constructed design from $G$, number of the blocks of the design, full automorphism group of the design and symmetric property of the design.

Example 2.1. In Table $2.1 D(G, M, n X)$ for some finite simple groups was constructed. By [4] in $L_{2}(11)$ centralizer of an element of class $2 A$ is maximal subgroup $D_{12}$. The design $D\left(L_{2}(11), D_{12}, 2 A\right)$ in row 3 of the table is a $1-(55,7,7)$ design with 55 blocks then is symmetric and $L_{2}(11)$ acts on the blocks primitively. This design is an example such that satisfies Lemma 2.2(1). In $G_{2}(3)$ for maximal subgroup $M=\left(3^{1+2} \times 3^{2}\right): 2 S_{4}$, consider $D\left(G_{2}(3), M, 3 A\right)$. Let $g \in 3 A$, in this case $\left|S_{g, M} \cap 3 A\right|=2$ and $\left|C_{G_{2}(3)}(g)\right|=\frac{|M|}{2}$ then $D\left(G_{2}(3), M, 3 A\right)$ in row 2 of the table is a $1-(364,13,13)$ symmetric design. This is an example that satisfies Lemma $2.2(2)$.

Always $t$-designs with $t \geq 2$ are interesting. In two following propositions we consider some conditions to construct $t$-designs for $t \geq 2$. 
Table 2.1: $D(G, M, n X)$ for some finite simple groups

\begin{tabular}{|c|c|c|c|l|c|c|c|}
\hline No. & $G$ & $M$ & $n X$ & $D(G, M, n X)$ & No. Blocks & $A u t(D)$ & Symm \\
\hline 1 & $A_{9}$ & $L_{2}(8): 3$ & $2 B$ & $1-(120,8,9)$ & 135 & $A_{9}$ & NO \\
\hline 2 & $G_{2}(3)$ & $\left(3^{1+2} \times 3^{2}\right): 2 S_{4}$ & $3 A$ & $1-(364,13,13)$ & 364 & $G_{2}(3)$ & YES \\
\hline 3 & $L_{2}(11)$ & $D_{12}$ & $2 A$ & $1-(55,7,7)$ & 55 & $L_{2}(11): 2$ & YES \\
\hline 4 & $S_{6}(2)$ & $2 .\left[2^{6}\right]:\left(S_{3} \times S_{3}\right)$ & $2 B$ & $1-(315,43,43)$ & 315 & $S_{6}(2)$ & YES \\
\hline 5 & $U_{5}(2)$ & $\left(2^{1+6} \times 3^{1+2}\right): 2 A_{4}$ & $2 A$ & $1-(165,37,37)$ & 165 & $U_{5}(2)$ & YES \\
\hline 6 & $U_{3}(3)$ & $4^{2}: S_{3}$ & $3 B$ & $1-(63,3,16)$ & 336 & $S_{6}(2)$ & NO \\
\hline 7 & $O_{8}^{+}(2)$ & $\left(3 \times U_{4}(2)\right): 2$ & $6 A$ & $1-(1120,8,360)$ & 50400 & $O_{8}^{+}(2)$ & NO \\
\hline 8 & $O_{8}^{+}(2)$ & $\left(3 \times U_{4}(2)\right): 2$ & $3 A$ & $1-(1120,40,40)$ & 1120 & $O_{8}^{+}(3): 4$ & YES \\
\hline
\end{tabular}

Proposition 2.3. Let $G$ be a finite simple group, $M$ be a maximal subgroup of $G$ and $\Omega$ be the set of all conjugates of $M$ in $G$. Let $n X$ be a conjugacy class of the elements of order $n$ such that $M \cap n X \neq \varnothing$ and $g \in n X$. Let $m \in\left\{1,2, \ldots, \chi_{M}(g)\right\}$. If intersection of every $m$ different conjugates of $M$ has $f \geq 1$ elements of the class $n X$ then $D(G, M, n X)$ is an $m-\left([G: M], \chi_{M}(g), \frac{f}{\left|S_{g, M} \cap n X\right|}\right)$ design. Also $G$ is an automorphism group of $D(G, M, n X)$ such that acts primitively on the points and transitively on the blocks of $D(G, M, n X)$.

Proof. Consider an $m$-set of different conjugates of $M$, set $S$ intersection of these subgroups. By the proof of Lemma 2.4(4) we have this partition $S \cap n X=$ $\cup_{j=1}^{h}\left(S_{g_{j}, M} \cap n X\right)$. Therefore for each $j \in\{1,2, \ldots, h\}$ these $m$ conjugates of $M$ are in a unique block. Then every $m$ conjugates of $M$ appears in exactly $\frac{|S \cap n X|}{\left|S_{g, M} \cap n X\right|}=\frac{f}{\left|S_{g, M} \cap n X\right|}$ blocks and result is concluded.

Example 2.2. In Table 2.2 we consider some finite simple groups in their 1-transitively action such that satisfy conditions of Proposition 2.3.

Table 2.2: Constructed $D(G, M, n X)$ from Proposition 2.3

\begin{tabular}{|c|c|c|c|l|c|c|c|}
\hline No. & $G$ & $M$ & $n X$ & $D(G, M, n X)$ & No. Blocks & Aut $(D)$ & Symm \\
\hline 1 & $S_{4}(3)$ & $3^{1+2}: 2 A_{4}$ & $3 \mathrm{~A}$ & $2-(40,13,4)$ & 40 & $L_{4}(3): 2$ & YES \\
\hline 2 & $S_{4}(4)$ & $2^{6}:\left(3 \times A_{5}\right)$ & $2 \mathrm{~A}$ & $2-(85,21,5)$ & 85 & $L_{4}(4): 2$ & YES \\
\hline 3 & $S_{4}(5)$ & $5^{1+2}: 4 A_{5}$ & $5 \mathrm{~A}$ & $2-(156,31,6)$ & 156 & $L_{4}(5): 4$ & YES \\
\hline 4 & $L_{2}(8)$ & $D_{18}$ & $2 \mathrm{~A}$ & $2-(28,4,1)$ & 63 & $L_{2}(8): 3$ & NO \\
\hline 5 & $L_{2}(16)$ & $D_{34}$ & $2 \mathrm{~A}$ & $2-(120,8,1)$ & 255 & $L_{2}(16): 4$ & NO \\
\hline
\end{tabular}

Corollary 2.1. Let $G$ be a finite simple group. Let $M$ be a maximal subgroup of $G$ and $\Omega$ be the set of all conjugates of $M$ in $G$. Let $n X$ be a conjugacy class of the elements of order $n$ such that $M \cap n X \neq \varnothing$ and $g \in n X$. For $t>1$ let the action of $G$ on $\Omega$ be $t$-transitive. For $m \in\{1,2, \ldots, t\}$ consider an $m$-set of different conjugates of $M$ and set $S$ intersection of these subgroups. If $S \neq\langle 1\rangle$ and $|S \cap n X|=k \geq 1$ then $D(G, M, n X)$ is an $m-\left([G: M], \chi_{M}(g), \frac{k}{\left|S_{g, M} \cap n X\right|}\right)$ design 
and $G$ is an automorphism group of $D(G, M, n X)$ that acts t-transitively on the points and transitively on the blocks.

Proof. Since $G$ is $t$-transitive on $\Omega$ then intersection of any $m$-set of different conjugates of $M$ is conjugate to $S$ and result is concluded by Proposition 2.3.

Example 2.3. In Table 2.3 we consider some finite simple groups in their 2-transitively action such that satisfy conditions of Corollary 2.1 .

Table 2.3: Constructed $D(G, M, n X)$ from Corollary 2.1

\begin{tabular}{|c|c|c|c|l|c|c|c|}
\hline No. & $G$ & $M$ & $n X$ & $D(G, M, n X)$ & No. Blocks & Aut $(D)$ & Symm \\
\hline 1 & $L_{2}(11)$ & $A_{5}$ & $2 \mathrm{~A}$ & $2-(11,3,3)$ & 55 & $L_{2}(11)$ & NO \\
\hline 2 & $L_{3}(3)$ & $3^{2}: 2 S_{4}$ & $2 \mathrm{~A}$ & $2-(13,5,15)$ & 117 & $L_{3}(3)$ & NO \\
\hline 3 & $L_{3}(3)$ & $3^{2}: 2 S_{4}$ & $3 \mathrm{~A}$ & $2-(13,4,1)$ & 13 & $L_{3}(3)$ & YES \\
\hline 4 & $L_{3}(5)$ & $5^{2}: G L_{2}(5)$ & $2 \mathrm{~A}$ & $2-(31,7,35)$ & 775 & $L_{3}(5)$ & NO \\
\hline 5 & $L_{3}(5)$ & $5^{2}: G L_{2}(5)$ & $5 \mathrm{~A}$ & $2-(31,6,1)$ & 31 & $L_{3}(5)$ & YES \\
\hline 6 & $S_{6}(2)$ & $U_{4}(2): 2$ & $2 \mathrm{~A}$ & $2-(28,16,20)$ & 63 & $S_{6}(2)$ & NO \\
\hline
\end{tabular}

\section{Constructed Designs from Sporadic Groups}

In this section we construct some designs from fourteen sporadic simple groups. For each considered sporadic group, we present one or two designs that their full automorphism groups are as the same sporadic group. These results are presented in Table 3.1. For information on the sporadic simple groups and their maximal subgroups we use Atlas [4].

In Table 3.1 the columns from left are: number of row, group $G$, maximal subgroup $M$ of $G$, a conjugacy class of $G$, properties of the constructed design from $G$, number of the blocks of the design, full automorphism group of the design and symmetric property of the design.

For instance we study properties of the designs in row 15 and 18 of Table 3.1.

The Conway group $\mathrm{Co}_{3}$ has order $495766656000=2^{10} .3^{7} .5^{3} .7 .11 .23$. The group $\mathrm{Co}_{3}$ has forty two conjugacy classes of elements and fourteen conjugacy classes of maximal subgroups. The centralizer subgroup of an elements of class $2 \mathrm{~A}$ in $\mathrm{Co}_{3}$ is maximal subgroup isomorphic to $2 . S_{6}(2)$. The group $M c L: 2$ is maximal subgroup of index 276 and $\mathrm{Co}_{3}$ acts 2-transitive on conjugates of $M c L: 2$. We consider design $D=D\left(C_{3}, M c L: 2,2 A\right)$. Intersection of every two different maximal subgroups conjugate to $M c L: 2$ have 2835 elements of class $2 A$ therefore by Corollary $2.1 D$ is a $2-(276,36,2835)$ design. The design $D$ has 170775 blocks and since centralizer of an element of the class $2 \mathrm{~A}$ is maximal hence by Proposition 2.1 group $\mathrm{Co}_{3}$ acts primitively on the blocks of this design. Also $\mathrm{Co}_{3}$ acts 2-transitively on the points of $D$. The full automorphism group of $D$ is $C o_{3}$. 
Table 3.1: Designs constructed from some sporadic groups

\begin{tabular}{|c|c|c|c|l|c|c|c|}
\hline No. & $G$ & $M$ & $n X$ & $D\left(M_{11}, M, n X\right)$ & No. Blocks & Aut $(D)$ & Symm \\
\hline 1 & $M_{11}$ & $L_{2}(11)$ & $2 \mathrm{~A}$ & $3-(12,4,3)$ & 165 & $M_{11}$ & NO \\
\hline 2 & $M_{11}$ & $2 . S_{4}$ & $2 \mathrm{~A}$ & $1-(165,13,13)$ & 165 & $M_{11}$ & YES \\
\hline 3 & $M_{12}$ & $M_{9}: S_{3}$ & $3 \mathrm{~A}$ & $1-(220,4,4)$ & 220 & $M_{12}$ & YES \\
\hline 4 & $M_{22}$ & $A_{7}$ & $4 \mathrm{~B}$ & $1-(176,4,315)$ & 13860 & $M_{22}$ & NO \\
\hline 5 & $M_{23}$ & $M_{22}$ & $3 \mathrm{~A}$ & $4-(23,5,16)$ & 28336 & $M_{23}$ & NO \\
\hline 6 & $M_{23}$ & $L_{3}(4): 2$ & $2 \mathrm{~A}$ & $1-(253,29,435)$ & 3795 & $M_{23}$ & NO \\
\hline 7 & $J_{1}$ & $2 \times A_{5}$ & $2 \mathrm{~A}$ & $1-(1463,31,31)$ & 1463 & $J_{1}$ & YES \\
\hline 8 & $J_{1}$ & $D_{6} \times D_{10}$ & $2 \mathrm{~A}$ & $1-(2926,46,23)$ & 1463 & $J_{1}$ & NO \\
\hline 9 & $J_{3}$ & $L_{2}(19)$ & $2 \mathrm{~A}$ & $1-(14688,96,171)$ & 26163 & $J_{3}$ & NO \\
\hline 10 & $H S$ & $U_{3}(5): 2$ & $2 \mathrm{~B}$ & $2-(176,12,66)$ & 15400 & $H S$ & NO \\
\hline 11 & $M c L$ & $M_{22}$ & $2 \mathrm{~A}$ & $1-(2025,105,1155)$ & 22275 & $M c L$ & NO \\
\hline 12 & $M c L$ & $2^{4}: A_{7}$ & $2 \mathrm{~A}$ & $1-(22275,435,435)$ & 22275 & $M c L$ & YES \\
\hline 13 & $H e$ & $2^{6}: 3 . S_{6}$ & $2 A$ & $1-(29155,651,558)$ & 24990 & $H e$ & NO \\
\hline 14 & $S u z$ & $U_{5}(2)$ & $3 \mathrm{~A}$ & $1-(32760,252,176)$ & 22880 & $S u z: 2$ & NO \\
\hline 15 & $C o_{3}$ & $M c L: 2$ & $2 A$ & $2-(276,36,2835)$ & 170775 & $C o_{3}$ & NO \\
\hline 16 & $F i_{22}$ & $O_{7}(3)$ & $2 A$ & $1-(14080,1408,351)$ & 3510 & $F i_{22}$ & NO \\
\hline 17 & $C o_{2}$ & $U_{6}(2): 2$ & $2 A$ & $1-(2300,284,7029)$ & 56925 & $C o_{2}$ & NO \\
\hline 18 & $F i_{23}$ & $2 . F i_{22}$ & $2 A$ & $1-(31671,3511,3511)$ & 31671 & $F i_{23}$ & YES \\
\hline
\end{tabular}

The Fischer sporadic simple group $F i_{23}$ has order $4089470473293004800=$ $2^{18} \cdot 3^{13} \cdot 5^{2} \cdot 7.11 .13 .17 .23$. The group $F i_{23}$ has ninety eight conjugacy classes of elements and fourteen conjugacy classes of maximal subgroups. The group $2 . F i_{22}$ is maximal subgroup of index 31671 and also is centralizer subgroup of an element of the class $2 A$. By Proposition 2.2(1) $D\left(F i_{23}, 2 . F i_{22}, 2 A\right)$ is a symmetric $1-(31671,3511,3511)$ design. The group $F i_{23}$ acts primitively on the points and the blocks of $D\left(F i_{23}, 2 . F i_{22}, 2 A\right)$. The full automorphism group of $D\left(F i_{23}, 2 . F i_{22}, 2 A\right)$ is isomorphic to $F i_{23}$.

\section{A 2-design invariant under $M_{22}$}

The Mathieu sporadic group $M_{22}$ has order $443520=2^{7} \cdot 3^{2} \cdot 5.7 .11$. The group $M_{22}$ has twelve conjugacy classes of elements and eight conjugacy classes of maximal subgroups [4].

Consider permutation representation of $M_{22}$ on 176 conjugates of maximal subgroup $A_{7}$. The group $M_{22}$ has one conjugacy class of elements of order 3 . The centralizer of an element of the class $3 A$ is isomorphic to $3 \times A_{4}$. Each elements of class $3 A$ is contained in 5 conjugates of maximal subgroup $A_{7}$.

Proposition 4.1. The $D\left(M_{22}, A_{7}, 3 A\right)$ is a 2-(176,5,4) design with full automorphism group isomorphic to $M_{22}$.

Proof. Let $M_{1}$ and $M_{2}$ be two different maximal subgroups isomorphic to $A_{7}$. Then $M_{1} \cap M_{2}$ is isomorphic to $3^{2}: 4$ or $S_{4}$. Both subgroups $3^{2}: 4$ and $S_{4}$ have 8 elements of order 3. Also for each $g \in 3 A, S_{g, A_{7}}$ is subgroup of order 3 . Hence by Proposition 
$2.3 \mathrm{D}\left(M_{22}, A_{7}, 3 A\right)$ is a $2-(176,5,4)$ design. The automorphism group is calculated by GAP [14].

Acknowledgement. The author thanks Dr. Ebrahim Ghorbani that computed automorphism groups of the designs from $\mathrm{Co}_{3}$ and $\mathrm{Fi}_{23}$ at Math. Computing Center of IPM (http://math.ipm.ac.ir/mcc).

\section{REFERENCES}

1. W. Bosma and J. J. Cannon: Handbook of Magma functions. School of Mathematics and Statistics, University of Sydney, Sydney (1995).

2. P. J. Cameron and A. Rudvalis: A design and a geometry for the group $F i_{22}$. Des. Codes Cryptogr. 44 (2007), no. 1-3, 11-14.

3. P. J. Cameron and J. H. van Lint: Designs, Graphs, Codes and their Links. Cambridge University Press, 1991.

4. J. H. Conway, R. T. Curtis, S. P. Norton, R. A. Parker and R. A. Wilson: An Atlas of Finite Groups. Oxford: Oxford University Press, 1985.

5. D. Crnković, V. Mikulić and B. G. Rodrigues: Designs, strongly regular graphs and codes constructed from some primitive groups. In Information Security, Coding Theory and Related Combinatorics (D. Crnkovic \& V. Tonchev, Eds.), IOS Press, Amsterdam, 2011, pages 231-252.

6. J.D. Dixon and B. Mortimer: Permutation Groups. Graduate Texts in Mathematics 163, Springer-Verlag, New York, 1996.

7. M.S. Ganief: 2-Generations of the Sporadic Simple Groups. PhD Thesis, University of Natal, 1997.

8. W.H. Haemers, C. Parker, V. Pless and V. D. Tonchev: A design and a code invariant under the simple group $\mathrm{Co}_{3}$. J. Combin. Theory Ser. A, 62 (2) (1993), $225-233$.

9. I.M. IsAacs: Character Theory of Finite Groups. Academic Press, San Diego, 1976.

10. J.D. KeY and J. MoorI: Designs, codes and graphs from the Janko groups $J_{1}$ and $J_{2}$. J. Combin. Math. and Combin. Comput., 40 (2002), 143-159.

11. J. Moori: Finite groups, designs and codes. Information security, coding theory and related combinatorics, 202-230, NATO Sci. Peace Secur. Ser. D Inf. Commun. Secur., 29, IOS, Am- sterdam, 2011.

12. J. Moori and B. G. Rodrigues: Some designs and codes invariant under the simple group $\mathrm{Co}_{2}$. J. of Algerbra, 316 (2007), 649-661.

13. J. Moori and B. G. Rodrigues: On some designs and codes invariant under the Higman-Sims group. Util. Math. 86 (2011), 225-239.

14. The GAP Team: GAP - Groups, Algorithms, and Programming. Version 4.5.5, 2012, (http://www.gap-system.org). 
Ali Reza Rahimipour

Faculty of Science

Department of Mathematics

University of Qom

P. O. Box 37161-46611

Qom, Iran

a.rahimipour@qom.ac.ir 